\title{
Design, Antropologia e Etnografia: pesquisa bibliográfica sobre a compreensão dos conceitos do Design Antropológico e Design Etnográfico no contexto brasileiro
}

Design, Anthropology and Ethnography: a bibliographic research about the comprehension of the concepts of Design Anthropology and Design Ethnography in the Brazilian scenario

SANTIAGO CAMINHA, Camille; Bacharel em Design; Universidade Federal de Pernambuco cnsc@cin.ufpe.br

SOUZA, Angélica; Bacharel em Design; Universidade Federal de Pernambuco; apcs2@cin.ufpe.br SANDRO GOMES, Alex; Prof. Doutor em Ciências da Educação, Universidade Federal de Pernambuco asg@cin.ufpe.br

ZILSE, Renata; Mestre em Design, Samsung Brasil, Campinas; renata.borges@samsung.com

\begin{abstract}
Resumo
Ao longo dos anos, o Design evoluiu em sua visão de prioridades, passando da intenção industrial para o "antropocentrismo projetual". Recentemente, a aproximação do Design com a Antropologia emerge como uma corrente com grande potencial. A metodologia Design Antropológico posiciona o usuário como elemento central e como participante ativo do processo de design. Compreendendo o potencial de aplicação dessa metodologia situada no Brasil, percebe-se a necessidade de entender os conceitos dos Design Antropológico e Design Etnográfico que ainda são nebulosos, prejudicando diretamente sua aplicação. Dessa forma, através de uma pesquisa na literatura internacional e nacional, este artigo possui o objetivo de embasar essas conceituações, além de compreender como os artigos acadêmicos brasileiros interpretam e utilizam essa abordagem.
\end{abstract}

Palavras Chave: Design Antropológico, Design Etnográfico, conceitos.

\begin{abstract}
Along the years, Design has changed in its vision of priorities, moving from the industrial intention to the "projectual anthropocentrism". Thus, the approximation between Design and Anthropology comes as a strong field, showing high potentials to application, that takes the user not just as a central and important element, but also as an active participant in the whole design process: the Design Anthropology methodology. Understanding the potential of applying this new situated methodology, in Brazil, the comprehension of the concepts and the distinction between Design Anthropology and Design Ethnography are nebulous, impairing directly its application. So, through a research in the national and international literature, this research aims to underpin these concepts, and also understand how the Brazilian academic articles interpret and use this approach.
\end{abstract}

Keywords: Design Anthropology, Design Ethnography, concepts. 


\section{Introdução}

O humano é um ser naturalmente criativo e detentor de capacidades físicas e cognitivas para adaptar a si mesmo e ao meio para sua sobrevivência. Dessa forma ele pôde evoluir desde o limar de pedras para caça, criando assim artefatos mais adequados para suprir suas necessidades, até a realidade tecnológica, em constante evolução, em que vivemos.

Desde os primórdios, os humanos dependem de sua criatividade e capacidade para entender e vencer as situações problemáticas e limitantes criando ferramentas mediadoras, melhorando sua qualidade de vida. Porém, com o advento industrial, as demandas cresceram de tal modo a afastar aqueles que ficaram responsáveis pelas criações da vivência e compreensão das suas reais necessidades dos consumidores.

Nos parágrafos a seguir, apresentamos historicamente, de forma simplificada, como o Design evoluiu em sua visão de prioridades passando da intenção industrial (despida de aproximação com o usuário) para o Design centrado no Usuário (DCU) e depois para o Design Centrado no Humano (DCH), no qual as pessoas tornam-se o elemento mais importante a ser estudado e inserido no processo de criação, proporcionando assim uma visão projetual "antropocêntrica".

Tomamos por início a apresentação de como o Design foi e é compreendido nos dias atuais. Surgiu entre o século XVII e fins do século XIX, quando "nasceu com o propósito de pôr ordem na bagunça no mundo industrial" (Cardoso, 2011, p.15). Nessa afirmação, Cardoso traz a compreensão de que o design emerge quando, pela primeira vez, o mundo lida com novas necessidades quantitativas de produção em grandes escalas. Nesse início, a priorização é focada para as indústrias na qual as produções devem ser econômicas, simples, eficientes e acima de tudo, lucrativas, introduzindo o mundo para artefatos essencialmente funcionais.

"A forma segue a função" foi uma máxima modernista que se popularizou pelo mundo iniciada na década de 20, junto à Escola Bauhaus e introduzida pelo arquiteto Sullivan. É nesse período que temos, na história da humanidade, a criação de objetos "desumanizados", tão simplificados em suas formas e valorizados apenas pela sua funcionalidade que perde suas conexões diretas com o homem, seja por meio da identificação estética ou por dificuldades no campo da usabilidade.

Porém, ainda que durante o período de prioridades, para a indústria, o Design não deixou de seguir o caminho da evolução. Com o passar do tempo, a atividade de projetar atinge um avanço no estudo de métodos e metodologias nos anos 1960 (Burdek, 2006, p.226). Essa característica indica a facilitação do ensinamento e replicação das atividades característicos de um designer. A evolução e expansão é potencializada e clara para o campo, apesar de que, nessa década o modelo funcionalista ainda era presente e as metodologias da época não contemplavam o estudo do usuário.

A gênese da introdução dos usuários na metodologia de design só surgiu no início dos anos 1970 e, apenas a partir da década de 1980, com a invenção do computador Macintosh, o usuário se tornou o centro dos projetos de fato. Com o início do advento tecnológico, as décadas de 1980 e 1990 marcaram um novo paradigma: a preocupação do usuário como centro de uma interação, impulsionado pela popularização dos computadores.

Dessa forma, esse paradigma juntamente com as novas tecnologias acarreta na necessidade 
de se reorientar e se readaptar as metodologias de design, abrindo as portas para os estudos envolvendo o usuário. Inicia-se então uma mudança significativa nas metodologias do campo, apresentando um distanciamento dos processos de projeto linear (problema- análise- solução) para se dedicar aos vários interesses e respectivas necessidades do usuário, em processos cíclicos e de retroalimentação. Segundo Burdek, (2006) essa nova vertente que se preocupa com o usuário final trouxe consigo novos temas para a área tais como usabilidade e design de interfaces, temas esses também relativos à ergonomia.

Da inserção do usuário como centro das interações e da ergonomia, surgiu o conceito de DCH (Giacomin, 2012). Dentre alguns pesquisadores que abordam esse conceito em suas produções acadêmicas destaca-se Klaus Krippendorff. Para o autor (Krippendorff, 2000), o DCH preocupa-se com a maneira que as pessoas vêem, interpretam e convivem com artefatos. Krippendorff (2007) sugere quatro pilares conceituais nos quais a abordagem do $\mathrm{DCH}$ deve ser sustentada. $\mathrm{O}$ primeiro pilar envolve o entendimento que o design é comprometido em projetar artefatos que vão ser experimentados por outros usuários de formas diferentes. O segundo pilar trata de projeto de artefatos que possibilite que os significados sejam apropriados pelo usuário. O terceiro pilar está relacionado aos interesses das pessoas envolvidas com a criação do artefato e, finalmente, o quarto pilar trata das interfaces no sentido da observação das práticas culturais dos usuários.

É a partir da necessidade de compreender o usuário, que se torna mais clara a aproximação do campo do Design (e o uso em suas metodologias) aos métodos utilizados pelas Ciências Sociais. Nesta pesquisa abordamos a aproximação especificamente com o campo da Antropologia e seus métodos de aproximação e compreensão de um grupo específico. No tópico a seguir, apresentaremos o porquê e como se deu essa aproximação historicamente (início até a atualidade), juntamente com o problema de pesquisa levantado neste estudo.

\section{Aproximação do Design e da Antropologia}

$\mathrm{Na}$ academia, já é clara a relação entre Design e Antropologia (Agar, 2014; Giaccardi, Cila, Speed, \& Caldwell, 2016; Pink, 2014; Rode, 2011; Segelstrom \& Holmlid, 2015) como duas áreas das Ciências Sociais que possuem potencial para congruência e criação de uma nova abordagem.

A Antropologia é uma área das Ciências Sociais que contempla métodos de cunho qualitativo para estudos de comportamentos de uma comunidade. Para Gunn et al. (2013, p.2) ela se caracteriza como um o estudo comparativo de sociedades e culturas, baseado em pesquisas empíricas detalhadas em contextos sociais concretos.

Seus métodos e técnicas são amplamente aplicados no processo de Design para compreensão dos comportamentos indo dos mais consolidados como observação, questionário, entrevista semi-estruturada, Focus Group e mapeamentos, aos mais recentes como video tour, video Re-enactment, Lurking entre outros que são muitas vezes mais exploratórios.

Junto à Antropologia, está a Etnografia. Para Gunn et al. (2013, p.2) o termo etnografia é ligado tanto ao processo de investigação, que significa também a imersão na vida social para compreendê-la e descrevê-la, quanto ao seu produto: a representação etnográfica final. A Etnografia pode ser composta por técnicas de observação, entrevistas, técnicas visuais e/ou tomadas de nota.

Na Indústria, a Etnografia também é utilizada há algumas décadas e possui uma aproximação com o Design no seu contexto histórico em relação a necessidade de entender o humano como 
centro mediador para concepção de softwares. Segundo Baba (1986), Schwartzman (1993), Reese (2002), (apud Gunn et al., 2013) essa aproximação teve início nos anos 1930, quando pesquisadores e designers colaboraram com antropólogos, no que foi o primeiro estudo a mostrar como os processos sociais informais afetaram a produção e a eficiência dos trabalhadores. $O$ autor também relata um acontecimento nos anos 1980, quando o Grupo de Prática e Tecnologia do Trabalho no Centro de Pesquisa Xerox Palo Alto (PARC) utilizou a pesquisa etnográfica em Design de Software (Gunn; Otto; and Smith, 2013). Para o autor, a partir dessa década, a Etnografia se tornou uma prática corrente para times interdisciplinares da área de computação, sendo, nos anos 1990, disseminada por outras equipes dos Estados unidos que foram inspiradas pelo trabalho do Centro de pesquisa Xerox.

Esse tratamento interdisciplinar continua na atualidade no qual muitas empresas multinacionais já compreendem como a Antropologia junto ao Design tem a capacidade de potencializar e aumentar as chances de sucesso em um projeto para o mercado. É na compreensão do público e inserção dos usuários reais no processo completo de criação que o Design se beneficia de modo a criar artefatos de verdadeiro valor mercadológico.

A metodologia que une a atividade dos designers com a visão dos antropólogos de uma forma adaptada às características de projetos de inovação, isto é, projetos que exigem maior eficiência e que se beneficiam do mergulho focado e rápido às situações específicas na realidade cotidiana, é conhecida como a metodologia do "Design Antropológico". Essa abordagem apresenta métodos para um mergulho profundo na compreensão dos usuários em suas características sociais, culturais e comportamentais, intitulado de "Design Etnográfico".

Essa pesquisa de campo focada, segundo Brereton et al. (2014), , aplica no Design, a "Etnografia Rápida" que consegue ser mais eficiente do que a etnografia tradicional encontrada na Antropologia e que mais rapidamente chegam a entendimentos que podem informar os profissionais de design com informações valiosas proporcionando oportunidades para insights.

O mesmo autor ainda afirma que enquanto o propósito da Etnografia é constantemente confundido pelos designers como apenas uma forma de coletar dados, a etnografia é um caminho poderoso para "abrir o jogo de possibilidades para o design" contribuindo para capacitar os designers a questionar os pressupostos incorporados na estrutura convencional de "problemasolução". (Brereton et al., 2014)

Mais adiante, será apresentado uma análise, na qual 19 artigos, entre publicações nacionais e internacionais, foram analisados com base nos conceitos que apresentavam sobre Design Antropológico e Design Etnográfico.

Essa compreensão da problemática envolvida nas produções acadêmicas nacionais veio a partir de uma pesquisa bibliográfica realizada previamente sobre a análise da compreensão do potencial da Antropologia para o Design nas produções acadêmicas no cenário brasileiro. Dessa forma, visto a problemática identificada, este artigo tem como objetivos apresentar o embasamento dos conceitos de Design Antropológico e Design Etnográfico, comparando-os com as definições da literatura internacional, trazendo também potenciais abordagens para aplicação em trabalhos futuros.

\section{Metodologia}

Foram pesquisados artigos com base em critérios pré-determinados para a escolha das 
amostras. A pesquisa exploratória internacional foi realizada em anais e congressos, nas bases ACM e SAGE, utilizando as palavras chave "anthropology", "ethnography" e "design" nos abstracts. Como o resultado encontrado não foi quantitativamente suficiente, foram utilizados para uma compreensão mais aprofundada sobre o tema alguns livros de autores que são referência na área.

A pesquisa nacional de cunho exploratório foi desenvolvida utilizando a ferramenta de pesquisa online específica para trabalhos acadêmicos, o Google Scholar, no intuito de encontrar os trabalhos no contexto nacional, utilizando as palavras-chave "antropologia", "etnografia" e "design" no título e conteúdo das publicações. Outro critério adotado foi a coleta de artigos publicados no espectro temporal dos últimos 10 anos cujo tema ou resumo estivesse relacionado com design antropológico e/ou design etnográfico.

Para análise dos dados, os seguintes campos foram identificados em cada artigo encontrado na pesquisa bibliográfica:

- $\quad$ a abordagem, no intuito de entender os temas que são abordados envolvendo as palavras chaves pesquisadas e o tipo de análise empregada, isto é, de bases teóricas ou aplicadas;

- definição de Design Antropológico, para analisar como as pesquisas brasileiras conceituam o campo do Design Antropológico, sendo esse um campo relativamente recente e ainda em desenvolvimento;

- definição de Design Etnográfico, para analisar como as pesquisas brasileiras conceituam a abordagem do Design Etnográfico;

- potencial para o design, no objetivo de entender como esses artigos compreendem quais as potencialidades de estudar e/ou aplicar os métodos do Design Etnográfico e Design Antropológico;

- exemplos de estudos, identificando qual os temas e objetivos dos contextos e exemplos empíricos abordados nos textos; e

- técnicas utilizadas, para identificar qual a variedade das técnicas que são aplicadas ou citadas nos estudos.

No presente trabalho, o foco aborda como os conceitos de Design Antropológico e Etnográfico são tratados na literatura internacional e analisa como essas áreas são compreendidas no cenário nacional brasileiro. Para análise e interpretação desses dados, foi utilizada a análise de temas principais, a fim de apontar os aspectos mais relevantes e compará-los para posteriores análises e conclusões.

\section{Conceituando Design Antropológico e Design Etnográfico segundo a literatura internacional}

Nesta seção apresentaremos primeiramente as compreensões que envolvem a abordagem antropológica e etnográfica junto ao Design encontradas na literatura internacional.

\subsection{Design Antropológico}

Para Gunn et al. (2013) Design Antropológico é um campo acadêmico de rápido desenvolvimento que combina elementos de Design e Antropologia. Complementando essa 
definição, Tunstall (2013) descreve que o Design Antropológico é proposto como uma metodologia que pode reformular tanto a Antropologia quanto a inovação do Design como práticas descolonizadas de engajamento cultural.

Essa abordagem une as teorias provindas do campo antropológico com os processos de criação do campo de inovação e criação do Design. Os praticantes do Design Antropológico seguem situações dinâmicas e relações sociais e estão preocupados com o "como as pessoas percebem, criam e transformam" seus ambientes através de suas atividades cotidianas. Essa visão desafia a ideia de que Design e inovação se referem apenas à criação de coisas novas como sendo centrais para processos de mudança social e cultural. As práticas do Design Antropológico ocorrem em diferentes escalas e cronogramas e envolvem muitas disciplinas, cada uma trazendo suas próprias maneiras distintas de conhecimento e de fazer. (GUNN, 2013)

Para finalizar, Gunn et al. (2013) ainda salienta que o Design e a Antropologia lidam com a oportunidade do designer trabalhar junto com o usuário e o impacto que isso traz na compreensão de objetos e produtos, sua criação e uso.

\subsection{Design Etnográfico}

Segundo Hooke (2012), a Etnografia no Design é um empreendimento muito diferente da etnografia nas ciências sociais: as responsabilidades são diferentes, as necessidades são diferentes, os resultados e seus usos são diferentes.

Design Etnográfico deve ser entendido como um método, utilizado no processo de Design, para compreender e imergir junto com o usuário no seu cotidiano, estudando o contexto real, não controlado, complexo e holístico, característicos de um determinado grupo ou região. Essa imersão deve buscar o entendimento de seus hábitos, dos padrões de uso, comportamentos, sentimentos, problemas e/ou situações, além de elementos invisíveis que acontecem nesse dia a dia. Suas técnicas podem compreender observação, entrevistas aprofundadas, técnicas visuais e de tomadas de nota, que serão adaptadas e combinadas de acordo com o contexto pesquisado.

Blomberg et al. (1993) ressaltam que a Etnografia não pode ser reduzida a um conjunto de métodos ou técnicas, ela deve ser entendida em relação aos seus princípios. Garfinkel (2012) também relatam que em um contexto de Design, o trabalho de campo e a etnografia são muitas vezes considerados a mesma coisa, o mesmo "método". Para o autor, embora a Etnografia exija trabalho de campo, isso não é tudo o que existe: a análise da organização intersubjetiva ou "social" da ação humana, incluindo o uso da tecnologia, também é necessária.

\subsection{Análise dos artigos encontrados na literatura internacional}

A análise a seguir é relacionada à amostra dos artigos internacionais encontrados na pesquisa bibliográfica exploratória e apresenta o conjunto de compreensões sobre o Design Antropológico e Etnográfico, que na maioria dos achados, abordam temas pontuais, mas, quando analisados em conjunto, apresentam um panorama que engloba as compreensões, entendimentos e possibilidades dessa abordagem.

\section{Design Antropológico e Design Participativo}

Um dos artigos encontrados do campo do IHC (Interação Humano Computador) intitulado "Reflexivity in Digital Anthropology" apresenta uma aplicação de projeto com caráter participativo, isto é, ele inclui o entendimento e importância do potencial de trazer o usuário para o processo de 
design.

Uma das vantagens (e desafios) dessa vertente, também conhecida como "Design Participativo", é a capacidade de incentivar pessoas a se envolverem no delineamento do futuro a partir das experiências vividas no passado e no presente. Tendo vivência real da situação, os participantes podem contribuir com propriedade, enfatizando os aspectos que thes são cruciais. (Canônica et al., 2014)

\section{A relação entre Design Antropológico e Design Etnográfico}

Junto ao Design Antropológico, se encontra o Design Etnográfico. A literatura internacional pesquisada aborda o Design Etnográfico como um método para imersão e compreensão do público, seus comportamentos e as particularidades do dia-a-dia, dando voz para os participantes que são os usuários finais. A seguir, será apresentado importantes citações identificadas nos artigos analisados sobre esses dois campos e suas relações.

Para Segelstrom \& Holmlid (2015), o Design Etnográfico evoluiu para uma abordagem própria, apropriando-se da prática etnográfica aplicadas para as necessidades e a realidade da prática de Design, com o propósito de "informar o Design". Nery \& Câmara (2015), falam que, no Design Etnográfico, o pesquisador vai além da observação e, ativamente, se envolve com as pessoas no campo com o intuito de inovar ou de modificar aquela realidade, utilizando ferramentas de design.

Van Veggel (2005, apud Segelstrom \& Holmlid, 2015) apresenta, pertinentemente, que a diferenciação entre Design Antropológico e Design Etnográfico deve ser compreendida abordando o Design Etnográfico como um método para entender o dia a dia das pessoas e o Design Antropológico como o entendimento em si. Para Blomberg et al. (1993), a Etnografia envolve a compreensão do mundo do ponto de vista dos estudados e os antropólogos tentam entender como as pessoas organizam seu comportamento e dão sentido ao mundo ao seu redor.

Assim, o Design Etnográfico deve ser entendido como um método de Design que engloba técnicas das mais clássicas como por exemplo "observação e entrevistas" até as mais novas como "Fly on the Wall", "Cultural Probes" e técnicas visuais que se utilizam de ferramentas como diários, anotações, fotografias, para imergir com o usuário e o Design Antropológico como uma metodologia para criação do entendimento. O quadro 1 a seguir apresenta os conceitos ordenados de acordo com as definições tradicionais de pesquisa e os achados nos artigos internacionais sobre métodos, técnicas e ferramentas.

Quadro 1 - Classificação dos conceitos encontrados na literatura internacional

\begin{tabular}{|c|c|c|c|}
\hline Metodologia & Método & Técnica & Ferramenta \\
\hline Design Antropológico & $\begin{array}{l}\text { Design } \\
\text { Etnográfico }\end{array}$ & $\begin{array}{l}\text { Observação; } \\
\text { Entrevista; } \\
\text { "Fly on the wall"; } \\
\text { "Cultural Probes"; } \\
\text { "Reenactment"; } \\
\text { "Tour"; "Behavioral mapping"; } \\
\text { etc }\end{array}$ & $\begin{array}{l}\text { Diário; } \\
\text { Fichas; } \\
\text { Fotografias; } \\
\text { Gravações em vídeo; } \\
\text { Post its; etc. }\end{array}$ \\
\hline
\end{tabular}


A Compreensão do potencial mercadológico da aplicação dos métodos etnográficos

O conceito relacionado à compreensão mercadológica da aplicação dos métodos etnográficos, com a finalidade de ampliar as possibilidades de sucesso em novos produtos a serem lançados, é apresentado por Segelstrom \& Holmlid, (2015), no momento em que eles apontam o Design Etnográfico como um modo de entender as particularidades do cotidiano e de aumentar o sucesso e reduzir falhas de um produto ou serviço causadas por uma falta de entendimento de comportamentos básicos.

\section{A interdisciplinaridade e o Design Antropológico}

Os artigos internacionais pesquisados trazem como fato importante no Design Antropológico a interdisciplinaridade de grupos e expertises de profissionais com conhecimentos que se complementam para o entendimento do cotidiano, observando aspectos culturais, e representando a voz das pessoas, característica notável e de grande importância, na medida em que possui a capacidade de potencializar sua aplicação, integrando conhecimentos de campos diversos com os do usuário.

\section{A temporalidade do Design Antropológico}

Entre os artigos encontrados dois são da professora Sarah Pink, doutora antropóloga, professora no Real Melbourne Institute of Technology na Austrália, e profissional altamente atuante no campo. Para Pink (2014), o diferencial trazido pelo Design Antropológico é a sua característica de modificar o futuro, possibilitando trabalhar em um mundo temporal em que o futuro é parte do que o designer procura intervir e modificar, por meio do entendimento das contingências e improvisações do presente e dos futuros desconhecidos, ou seja, fornecendo a possibilidade de uma visão do que pode ser (futuro), com base no que foi (passado) e no que é (presente).

A seguir, os próximos tópicos de análise são referentes aos temas encontrados na amostra brasileira. Nessa fase, a observação dos temas será comparada às análises dos artigos internacionais.

\section{Conceituando Design Antropológico e Design Etnográfico segundo a literatura nacional brasileira}

Avançando a pesquisa, a análise agora é relacionada à amostra dos artigos nacionais encontrados na pesquisa bibliográfica exploratória e objetiva entender como o campo acadêmico de Design brasileiro entende a vertente antropológica aplicada para o desenvolvimento de projetos.

Serão apresentadas a amostra, as abordagens e os tipos de pesquisas, além dos temas gerais para serem triangulados com os achados na literatura internacional.

\section{Amostra, abordagem e tipo de pesquisa}

Foram pesquisados treze artigos brasileiros, sendo quatro do Congresso Brasileiro de Pesquisa e Desenvolvimento em Design (P\&D); e apenas um das demais fontes: Revista E-LOGO, Colóquio Internacional de Design, InfoDesign, revista Arcos Design, Design Research Journal, Revista de Literatura, Linguística e Artes; revista E-Tech: Tecnologias para Competitividade Industrial, Colóquio Internacional de Design e anais do Seminário Integrado de Software e Hardware (SEMISH).

As áreas de pesquisas desses artigos englobam Design (sete artigos), Empreendedorismo (três artigos), Interação Humano Computador - IHC (dois artigos) e Linguística e Artes (um artigo). 
Os trabalhos pesquisados foram tanto de caráter teórico/bibliográfico (cinco artigos), quanto de caráter empírico/experimentais (oito artigos). Dentre alguns dos trabalhos teóricos, as conceituações e debates foram apresentados de forma consistente, porém vale salientar que, apesar de mais da metade dos artigos encontrados serem de caráter empírico, suas conceituações para os campos abordados foram superficiais e a aplicação prática ainda é limitada.

Em relação a abordagem dos artigos, alguns dos trabalhos nacionais também tratam da relação entre Design e Antropologia e abordam a Etnografia junto ao Design, sendo quatro da área de Design, um da área de IHC e três da área de Empreendedorismo. Dentre esses artigos, oito abordam o conceito de etnografia, porém apenas quatro conceituam o Design Etnográfico, diferenciando sua forma de aplicação da Etnografia tradicional e dos três artigos encontrados que discorrem sobre o Design Antropológico, dois não fazem referência à etnografia.

No geral, é perceptível a compreensão sobre o potencial que há na aproximação do Design com as áreas das Ciências Sociais, mesmo que as abordagens entre os artigos sejam levemente diferenciadas. Apesar de que nenhum dos trabalhos empíricos na amostra brasileira falam sobre projetos inovativos ou visão de futuro, foi identificado três diferentes caminhos de interesse para a adoção da abordagem antropológica: 1) a valorização cultural, 2) o desenvolvimento de projetos para grupos populacionais complexos e delicados ou 3) o desenvolvimento de soluções para problemáticas atuais e imediatas.

\section{Relação com o Design Participativo}

Dos treze artigos pesquisados, seis abordam a metodologia do Design Participativo, onde três discorrem sobre Design e Antropologia e os outros três sobre Design e Etnografia.

Apesar de o Design Participativo estar presente em seis artigos, e essa abordagem ser de caráter tanto teórico quanto empírico, grande parte dos trabalhos apresentam esse conceito através de um olhar cultural e intervenção no contexto social.

Dos artigos que tratam sobre Design Antropológico, Anastassakis, (2014) e Mota \& Sirito, (2016) relacionam o papel da Antropologia a um processo participativo, em um procedimento colaborativo de imersão que une pesquisadores e designers junto aos usuários durante todo o processo de Design. No entanto, apenas um dos artigos apresenta claramente o conceito relacionado com a criação de futuros colaborativos, mesmo que não seja relacionado à área de inovação tecnológica.

\section{Design Etnográfico para imersão}

Da amostra pesquisada, os artigos que tratam do Design Antropológico e do Design Etnográfico reconhecem o uso da metodologia e do método para imersão no comportamento, cotidiano, situações, atividades, necessidades e contextos culturais dos usuários reais, isto é, informações para uma compreensão de forma holística em congruência com as conceituações encontradas na amostra internacional.

Também é descrita a relação dessa imersão com o processo criativo de Design tanto na relação de construção de objetivos e requisitos, quanto na proposição de intervenções bem como uma forma de adquirir uma vantagem competitiva.

\section{Objetivo de compreensão do contexto e valorização cultural e artística}

$\mathrm{Na}$ amostra encontrada, oito artigos apresentam o uso do Design Antropológico, Design 
Etnográfico e Etnografia com o objetivo de entender o contexto cultural e, dentre esses, em três publicações (Anastassakis, 2014; Nery et al., 2015; Perpétuo \& Noronha, 2016) é evidente o uso da abordagem Antropológica como forma de valorização da cultura.

Sobre os achados: quatro artigos abordam o Design Antropológico, o Design Etnográfico e a Etnografia como forma de intervenção no ambiente, provocando impacto no território; três abordam o potencial cultural da pesquisa etnográfica, tornando possível ao pesquisador descobrir o sistema de significados culturais, seu desenvolvimento e sua influência; e um traz a interessante abordagem ao contexto brasileiro que é o uso de métodos antropológicos e etnográficos no Design, com o objetivo da valorização cultural. Através das diferentes culturas nacionais, esses métodos são utilizados em alguns artigos para a criação e análise de projetos de essência cultural, característica de resultados antropológicos.

Apesar de a literatura internacional trazer o caráter intervencionista do Design Antropológico, a literatura nacional apresenta esse conceito através de uma proposta social. Nos artigos brasileiros, o Design Antropológico e o Design Etnográfico é aplicado e descrito como forma de intervenção em comunidades, descobrindo significados culturais característicos da diversidade brasileira, onde dois desses trabalhos tem como resultado final a criação de artefatos de caráter e de identidade cultural.

Esse uso específico, sensato e de grande relevância da abordagem antropológica para criação de artefatos culturais, como forma de valorizar fortes referências culturais locais, foi uma das características mais singulares encontrada nessa amostra e a razão para esse determinado tipo de projeto pode ser atribuído à ampla extensão territorial e à grande diversidade cultural presentes no Brasil, onde essa multiplicidade pode e deve ser divulgada e exaltada. Esse tipo de aplicação não foi encontrado na amostra internacional e aqui levanta-se duas hipóteses: essa falta pode ser devido às bases de pesquisa serem de teor tecnológico ou ser uma forma de aplicação característica brasileira que visa valorizar sua multiplicidade cultural, abrindo portas para maiores pesquisas.

\section{Etnografia para inovação}

$\mathrm{Na}$ amostra pesquisada, três artigos teóricos, fazem uma relação entre Etnografia e inovação. Um dos artigos foi publicado no Colóquio Internacional de Design, o segundo foi publicado na revista E-Logo e o terceiro na E-Tech- Tecnologia para Competitividade Industrial. As duas revistas encontradas envolvem o tema empreendedorismo, inovação e competitividade. Dos três artigos, um relaciona inovação à Etnografia Rápida, trazendo seu uso para a criação de cenários; outro relaciona o uso da Etnografia para obter insights e o terceiro traz a etnografia como técnica de reconhecimento de oportunidades de inovação.

Visto que apenas dois dos artigos brasileiros encontrados nas fontes da área de Design abordaram temas sobre o desenvolvimento de inovações tecnológicas para o futuro, contrariando a abordagem do restante da amostra que trabalha basicamente no campo temporal da atualidade (para valorização cultural ou solução de problemáticas imediatas), isso pode indicar que o Design Antropológico e Etnográfico ainda está muito centrado no mundo acadêmico, sem necessariamente ser aplicado em projetos de mercado, tecnologicamente inovativos, temas esses propostos e debatidos nos estudos teóricos encontrados na fontes de Empreendedorismo.

Essa falta de trabalhos (principalmente de âmbito prático) que abordam a inovação para cenários futuros vai de encontro com os achados internacionais que entendem, apresentam e 
valorizam o Design Antropológico para criação de novas realidades tecnológicas indicando uma diferente inclinação de interesse relativos aos trabalhos nacionais.

Outro fator que vale destacar é que, apesar de alguns artigos apresentarem uma correlação da Etnografia com a criação de tecnologias diferenciadas, eles não falam do contexto de inovação para tecnologias disruptivas. Dessa amostra, somente os artigos que relacionam etnografia e inovação, que são de caráter teórico, fazem essa ligação, apenas apresentando exemplos de casos internacionais.

Em relação aos artigos teóricos, somente um apresenta claramente o conceito do Design Antropológico relacionado com a criação de futuros colaborativos. Diante desse fato, a amostra pesquisada leva a sugerir que o Design Antropológico, em relação a criação de futuros colaborativos, no Brasil, ainda pode ser incipiente.

\section{Análise comparativa entre as amostras internacionais e nacionais}

Compreendendo a aplicação dos conceitos de Design Etnográfico e Design Antropológico no cenário nacional e no cenário internacional a partir da amostra de artigos pesquisada, a seguir será apresentada uma comparação entre essas compreensões e sugestões para possíveis pesquisas futuras. Essa análise comparativa está exposta por temas introdutórios e pontos para reflexão.

\section{Design Antropológico e o que é bem compreendido}

Os artigos brasileiros que tratam sobre Design Antropológico o fazem de acordo com conexões encontradas na literatura internacional. Três artigos apresentam congruências na questão da interdisciplinaridade: (Anastassakis, 2013, 2014; Mota \& Sirito, 2016) e um dos artigos fala indiretamente da temporalidade do Design Antropológico (Mota \& Sirito, 2016).

Um dos artigos da professora Anastassakis aborda o Design Antropológico, através de teorias de Tim Ingold, como um exercício de experimentação interdisciplinar que busca operar a partir de uma conjugação dialógica entre Design e Antropologia (Anastassakis, 2014). Outra abordagem interessante é do artigo de Mota \& Sirito. Este é rico e consistente em seus argumentos, abordando o Design Antropológico de acordo com os conceitos encontrados na literatura internacional. É interessante também que os autores utilizam de citações da autora Anastassakis.

Apesar de não estar diretamente explícita nos artigos nacionais, a capacidade do Design Antropológico de entender relações temporais, corrente essa presente na literatura internacional e apresentada por pesquisadores como Gunn, Otto, Pink, entre outros, já se encontra presente em um artigo nacional (Mota \& Sirito, 2016) , mesmo que de forma teórica falando que os praticantes do Design Antropológico estão preocupados como as pessoas percebem, criam e transformam seus ambientes e deixando claro que o seu uso também serve para facilitar o fazer de futuros colaborativos.

Essa característica temporal possui um potencial para pesquisas de Design no cenário nacional, tanto no âmbito dos métodos e técnicas de Design, quanto na aplicação destes para intervenções urbanas, sociais, criação de valor e inovação.

\section{Design Antropológico, Design Etnográfico e o processo de Design}

$\mathrm{Na}$ amostra nacional, vale destacar que, no geral e de maneira congruente, é evidenciado o entendimento e uso do método etnográfico, aplicado durante a fase de exploração do problema, para a compreensão dos comportamentos e situações próprias do grupo analisado, diferenciando 
entre os trabalhos publicados apenas em relação ao tempo de aplicação do método.

Porém, tanto o Design Etnográfico como a metodologia do Design Antropológico podem e devem ser utilizados nas várias fases do processo completo de Design. O Design Antropológico engloba um processo de desenvolvimento que não valoriza apenas a compreensão profunda dos usuários finais, como também traz a presença desses usuários em fases de criação e testes situados em ambientes reais em uma dinâmica de aprendizado mútuo. Como exemplos têm-se os artigos internacionais que trazem o uso do Design Etnográfico (Giaccardi et al. 2016) (Pink, 2014) para compreender o dia a dia do usuário; e o Design Antropológico para desenvolvimento e criação através de workshops participativos, observando o conceito de temporalidade e ferramentas de Design (Pink, 2014) (Selgestrom \& Holmlid, 2015].

Outro ponto interessante para análise é a percepção de que a aplicação conjunta e complementar do Design Participativo com o Design Antropológico é uma característica igualmente encontrada nos artigos internacionais, indicando que são projetos que vão além da imersão e compreensão do público, incluindo-os igualmente no processo de criação das ideias propostas.

\section{Design Antropológico e Design Etnográfico e as técnicas utilizadas}

Em relação às técnicas aplicadas dos artigos que possuem uma abordagem empírica, as práticas estão em sua maioria restritas a técnicas de observação e entrevistas, quando comparadas a variedade das técnicas utilizadas internacionalmente. Na literatura internacional, muitas técnicas de design etnográfico são adaptadas a partir das existentes, resultando em uma variedade para aplicação em diferentes contextos. Algumas variações de técnicas citadas entre os artigos internacionais foram as: cultural probes $^{1}$, magic thing ${ }^{2}$ e thing ethnography ${ }^{3}$ (Giaccardi et al., 2016; Segelstrom \& Holmlid, 2015).

\section{Nebulosidade em reação a compreensão dos conceitos de Etnografia}

Apesar de existir a compreensão em relação a aproximação entre as áreas e o potencial do Design Antropológico e do Design Etnográfico para o Design, a grande parte da amostra na literatura nacional não traz um entendimento desses conceitos de forma clara, principalmente nos trabalhos aplicados empiricamente, como acontece na literatura internacional, além de não deixar explícita a diferença entre Design Antropológico e Design Etnográfico, dificilmente utilizando os dois conceitos no mesmo artigo. É identificável também que existe a compreensão do uso de uma "Etnografia adaptada" para o campo do desenvolvimento, sem necessariamente chamá-la de "Design Etnográfico".

Porém, apesar da compreensão sobre a Etnografia, os artigos pesquisados apresentam problemas na compreensão geral em relação a etnografia, referenciando-a em diferentes artigos como uma metodologia, método ou técnica, criando uma confusão para o leitor na compreensão desses conceitos acadêmicos. Os dois artigos que tratam de Etnografia (um na área de IHC que fala

\footnotetext{
1 'As "cultural probes", então e agora, são tipicamente um pacote com material, adereços e instrumentos que permitem que um informante documente o dia a dia de maneiras específicas. Diários, câmeras, mapas, sugestões de atividades, ferramentas e questionários visuais, cartões postais, etc. são frequentemente encontrados nesses pacotes.

${ }^{2} \mathrm{~A}$ "magic thing" é uma peça em branco que é introduzida em uma situação de pesquisa do usuário. O informante deve usar este suporte para atingir seus objetivos e o estudo é feito com técnicas etnográficas.

3 A perspectiva de uma "thing" que está ativamente situada dentro das relações com outras entidades, e que tem diferentes trajetórias que os humanos, pode apresentar novos caminhos para humanos e não-humanos.
} 
de Design Etnográfico e outro na área de empreendedorismo que fala sobre pesquisa etnográfica e inovação), abordam o Design Etnográfico como uma metodologia. Há também artigos que não atentam para a diferenciação do que seria uma técnica etnográfica e uma ferramenta. Um artigo usa o termo ferramentas ao invés de técnicas para entrevistas, questionários e a coleta de histórias.

Alguns artigos também não compreendem os princípios do que seria um método etnográfico, englobando nesse método, por exemplo, métodos tradicionais do Design como Grupo Focal, onde a pesquisa não é realizada no contexto real (Blomberg et al., 1993), e descrevendo algumas técnicas etnográficas como estudos de campo, análise contextual, estudo observacional e observação participante, como sinônimos de etnografia, relacionando o termo Etnografia a uma técnica.

\section{A Compreensão do potencial mercadológico dos métodos etnográficos}

Tanto nos artigos nacionais quanto nos artigos internacionais, os métodos etnográficos são compreendidos como métodos para inovar, apesar de que, na literatura nacional, essa compreensão só é apresentada em trabalhos teóricos, onde um deles descreve estudos de casos internacionais relativos à empresas que aplicaram o Design Antropológico para dar força à sua argumentação.

Outra questão que a literatura nacional traz, presente em um artigo da amostra pesquisada e que é consolidado na literatura internacional, é a capacidade do Design Etnográfico de identificar comportamentos mundanos que podem revelar fatos surpreendentes. Essas situações podem trazer disrupções, tanto na criação de artefatos e serviços inovadores, quanto na questão da intervenção urbana e valorização cultural.

\section{Considerações finais: aplicando o Design Antropológico - Potencial para futuros trabalhos no contexto nacional}

A aproximação entre o Design e a Antropologia é algo bem estabelecido na literatura internacional, porém, como visto na pesquisa bibliográfica, ainda cursa um caminho de desenvolvimento na literatura nacional. Apesar disso, ambos contextos brasileiro e internacional trazem a essência da relação interdisciplinar entre as áreas presente tanto na junção de teorias quanto na aplicação em equipes de criação e desenvolvimento.

Apesar dessa compreensão, em alguns artigos na amostra da literatura brasileira os conceitos sobre Design Antropológico e Design Etnográfico apresentam-se nebulosos e confusos. A comparação realizada levou a perceber que ainda são necessários trabalhos que conceituem o Design Antropológico e o Design Etnográfico de forma mais clara, diferenciando e estabelecendo os limites entre a metodologia Design Antropológico, o método Design Etnográfico, as técnicas etnográficas e as ferramentas que podem ser utilizadas em um experimento que adote essa metodologia. Outra questão que deve ser considerada nos trabalhos nacionais é da abordagem antropológica e dos métodos etnográficos nas várias fases do processo de Design, não os restringindo à fase de exploração de problemas.

É também observada a necessidade de trabalhos de Design empíricos de cunho nacional que entendam os conceitos de forma clara e apliquem em seus estudos o Design Antropológico e o Design Etnográfico de forma coerente. 
Uma interessante hipótese que surgiu desse trabalho e que pode ser importante para pesquisas futuras é entender se o Design Antropológico e o Design Etnográfico, utilizado em projetos sociais para valorização cultural, pode ser uma característica brasileira ou de países que possuam algum grau de diversidade cultural, ou se isso é algo comum na literatura de Design internacional e o entendimento disso nessa pesquisa foi limitado pela amostra pequena e de base tecnológica nos artigos internacionais.

Outro potencial para pesquisas no Brasil é o uso do Design Antropológico e do Design Etnográfico para projetos na área inovação. Enquanto os artigos nacionais pesquisados trouxeram essa questão de forma mais teórica, a literatura internacional de Design possui projetos aplicados, já há algumas décadas, e a inovação proporcionada pelo uso da abordagem antropológica é algo já estabelecido. Além disso, a temporalidade característica do Design Antropológico na literatura internacional é também aplicada em projetos inovadores que contemplam a compreensão do cotidiano do usuário. Sendo assim, utilizar o Design Antropológico e o Design Etnográfico, no cenário nacional que traz diversidade e identidade cultural, pode ser um rico ponto de partida para inovações e intervenções disruptivas na concepção de artefatos digitais e sociais, na comunidade e no ambiente urbano.

\section{Referências}

Agar, M. (2014). An outsider's ethnographic thoughts about design. Arts and Humanities in Higher Education, 0(0), 1-8. https://doi.org/10.1177/1474022214531478

Alves, C. M., Prado, G., Lopes, P. G., \& Gomez, L. S. R. (2017). Poéticas na arte, no design e na antropologia : uma análise de dois projetos artístico-fotográficos Poetics in art, design and anthropology : an analysis of two artistic- photographic projects. Revista de Literatura, Linguística $E$ Artes, v. 13, , 180-200.

Anastassakis, Z. (2014). Design e Antropologia: considerações teóricas e experimentações práticas em diálogo com a perspectiva do antropólogo Tim Ingold. Blücher Design Proceedings.

Anastassakis, Z. (2013). Laboratório de Design e Antropologia : preâmbulos teóricos e práticos. Arcos Design, 7(1), 178-193.

Blomberg, J., Giacomi, J., Mosher, A., \& Swenton-Wall, P. (1993). Ethnographic Field Methods and Their Relation to Design. In Participatory Design: Principles and Practices (pp. 123-155). Hillsdale, NJ, USA: L. Erlbaum Associates Inc. Hillsdale. https://doi.org/10.1007/BF01305846

Burdek, B. E. (2006). História, Teoria e Prática do Design de Produtos. São Paulo: Editora Edgard Blücher.

Canônica, R., Peixe, R. I. P., Santos, A. S., \& Kohls, C. (2014). Relações entre o Design Participativo e princípios pedagógicos Freireanos. In P\&D Design (Vol. 1, pp. 1-12). Gramado- Rio Grande do Sul.

Cardoso, R. (2011). Design para um mundo complexo. São Paulo: Cosac Naify.

Crabtree, A., Rouncefield, M., \& Tolmie, P. (2012). Doing Design Ethnography. In Human-Computer Interaction Series (p. VIII, 208). London: Springer-Verlag London. https://doi.org/10.1007/978-14471-2726-0

Giaccardi, E., Cila, N., Speed, C., \& Caldwell, M. (2016). Thing Ethnography. Proceedings of the 2016 ACM Conference on Designing Interactive Systems - DIS '16, 377-387. 
https://doi.org/10.1145/2901790.2901905

Giacomin, J. (2012) What is Human Centred Design?. In: X Congresso Brasileiro de Pesquisa em Design, São Luís - MA, Anais... São Luís: EDUFMA,, p.148-161.

Gunn, Wendy; Otto, Ton; and Smith, R. C. (2013). Design Anthropology: Theory and Practice. Bloomsbury.

Krippendorff, K; Butter, R.. (2007) Semantics: Meanings and Contexts of Artifacts. In SCHIFFERSTEIN, H.N.J.; HEKKERT, P. (Eds.). Product experience. New York: Elsevier.p.353-376.

Kripperndorff, K.(2000).Propositions of Human-centeredness: A Philosophy for Design. In: Durling, D.; Friedman, K. (Eds.). Doctoral Education in Design: Foundations for the Future. Staffordshire (UK): Staffordshire University Press. p.55-63.

Lucca, A. de S. (2016). A etnografia rápida no metaprojeto de design para o território. E-Revista LOGO, v.5, n.1, 23-36.

Massami Watanabe, W., \& Fortes, R. P. de M. (2012). Revisão Sistemática sobre princípios de design de aplicações web acessíveis para analfabetos funcionais. Anais Do Seminário Integrado de Software E Hardware (SEMISH), 403-417.

Meyer, G., \& Damazio, V. (2012). Notas de uma etnografia do Design. Strategic Design Research Journal, 5(3), 120-128. https://doi.org/10.4013/sdrj.2012.53.04

Mota, C., \& Sirito, M. (2016). Design em movimento: repensando o design através do encontro com as ciências sociais. Blücher Design Proceedings, 9(num. 2). Retrieved from proceedings.blucher.com.br

Nery, C. G., Magalhães, L. A. C., Câmara, J. J. D., \& Lana, S. L. B. (2015). Os livros digitais de fotografia da coleção festejo maior: nexos possíveis entre design, etnografia e antropologia visual. Blücher Design Proceedings.

Perpétuo, N. C. F., \& Noronha, R. G. (2016). Estar lá, mas também do outro lado da tela: limites e possibilidades da etnografia em ambientes virtuais e a percepção de blogueiras de moda sobre design, produtos e sustentabilidade. Blücher Design Proceedings.

Pink, S., Mackley, K. L., Mitchell, V., Hanratty, M., Escobar-Tello, C., Bhamra, T., \& Morosanu, R. (2008). Applying the lens of sensory ethnography to sustainable $\mathbf{H C l}$. ACM Transactions on Computer-Human Interaction, 20(4), 1-18. https://doi.org/10.1145/2494261

Pink, S. (2014). Digital-visual-sensory-design anthropology: Ethnography, imagination and intervention. Arts and Humanities in Higher Education, 13(4), 412-427. https://doi.org/10.1177/1474022214542353

Rode, J. a. (2011). Reflexivity in Digital Anthropology. Chi 2011, 1-10. https://doi.org/10.1145/1978942.1978961

Santa Rosa, J. G., Gurgel, A., \& Passos, M. (2012). Técnicas Baseadas em Etnografia e Prototipagem no Design de interface de Aplicativo Mobile para Gerenciamento Acadêmico. InfoDesign, 9(2), 8899. Retrieved from http://www.infodesign.org.br/revista/index.php/infodesign/article/viewFile/123/94

Segelstrom, F., \& Holmlid, S. (2015). Ethnography by design: On goals and mediating artefacts. Arts 
and Humanities in Higher Education, 14(2), 134-149. https://doi.org/10.1177/1474022214560159 Tarachucky,L. , \& Gomez, L. S. R. (2013). Pesquisa etnográfica aplicada a processos de inovação Estado da arte e perspectivas. Colóquio Internacional de Design, (Edição 2013), 2-8.

Tavares, P., Agner, L., \& Ferreira, S. (2010). Observações Etnográficas na Avaliação da Usabilidade de Dispositivos Móveis de Coleta de Dados Estatísticos. Interaction South America, (April 2016). Retrieved from http://www.agner.com.br/download/artigos/Artigo - Congresso Interaction SA 2010.pdf

Toé, R. D. A. D., Belo, J. A., \& Tosta, H. (2011). A pesquisa etnográfica como estratégia para inovação. Revista E-Tech: Tecnologias Para Competitividade Industrial, 4(1), 68-81. Retrieved from http://revista.ctai.senai.br/index.php/edicao01/article/view/176 